\title{
1,8-bis((S)-4-tert-butyl-4,5-dihydrooxazol-2-yl)-3,6-diphenyl-9H-carbazole (1c)
}

$\mathrm{CuCN}$ (0.268 g, $2.99 \mathrm{mmol})$ was added to a solution of 1,8-diiodo-3,6-diphenyl carbazole (2) (0.605 g, $1.06 \mathrm{mmol})$ in DMF (15 ml) under Ar atmosphere, and the resulting mixture was refluxed for $1.5 \mathrm{~h}$. The reaction mixture was cooled to ambient temperature, filtered to remove precipitate, and the filtrate was poured into $3 \%$ aqueous $\mathrm{NaCN}(100 \mathrm{ml})$. The precipitated solid was partitioned between ethyl acetate $(300 \mathrm{ml})$ and a solution of ethylenediamine $(3 \mathrm{ml})$ in $\mathrm{H}_{2} \mathrm{O}(100 \mathrm{ml})$, and the organic layer was washed with $\mathrm{H}_{2} \mathrm{O}(50 \mathrm{ml})$, then brine $(50 \mathrm{ml})$, dried over $\mathrm{Na}_{2} \mathrm{SO}_{4}$. Evaporation of the solvent and drying under vacuum for 1 day afforded crude 1,8-dicyano-3,6-diphenyl carbazole. This crude product was suspended in $\mathrm{PhCl}(15 \mathrm{ml})$, and to this suspension were added L-tert-leucinol $(0.375 \mathrm{~g}, 3.20 \mathrm{mmol})$ and $\mathrm{ZnCl}_{2}$ (0.402 g, $\left.2.95 \mathrm{mmol}\right)$ under Ar. The reaction mixture was refluxed for $65 \mathrm{~h}$, and then cooled to ambient temperature. After the addition of a solution of ethylenediamine ( $3 \mathrm{ml})$ in $\mathrm{H}_{2} \mathrm{O}(20 \mathrm{ml})$, the mixture was stirred for $1 \mathrm{~h}$. The resulting mixture was extracted with $\mathrm{CH}_{2} \mathrm{Cl}_{2}(20 \mathrm{ml}$ x 4), and the combined organic layer was washed with brine $(50 \mathrm{ml})$, dried over $\mathrm{Na}_{2} \mathrm{SO}_{4}$, evaporated. The resulting orange viscous oil was purified by flash chromatography (hexane/ $\mathrm{CH}_{2} \mathrm{Cl}_{2}=4 / 1-1 / 1$ ). Recrystallization from hexane/ethyl acetate afforded a pale yellow crystal $\mathbf{1 c}(93 \%, 2$ steps from 2$)$ : $[\alpha]_{D}{ }^{24}+108$ (c 1.16, $\mathrm{CHCl}_{3}$ ); mp 230-231 ${ }^{\circ} \mathbf{C} ;{ }^{1} \mathrm{H}$ NMR (400 MHz, $\mathrm{CDCl}_{3}$ ): $\delta=11.9$ (s, 1H), 8.47 (d, $\left.J=1.7 \mathrm{~Hz}, 2 \mathrm{H}\right), 8.22$ (d, $J=1.7 \mathrm{~Hz}, 2 \mathrm{H}$ ), 7.76 (dddd, J=7.3, 1.7, 1.7, $1.2 \mathrm{~Hz}, 4 \mathrm{H}$ ), 7.49 (dddd, J=7.3, 7.3, 1.7, $1.2 \mathrm{~Hz}, 4 \mathrm{H}$ ), 7.36 (tt, $J=7.3,1.7 \mathrm{~Hz}, 2 \mathrm{H}), 4.42$ (dd, J=9.9, $8.5 \mathrm{~Hz}, 2 \mathrm{H}), 4.36$ (dd, $J=8.5,6.8 \mathrm{~Hz}, 2 \mathrm{H}), 4.22$ (dd, J=9.9, $6.8 \mathrm{~Hz}, 2 \mathrm{H}), 1.04$ (s, 9H); ${ }^{13} \mathrm{C} \mathrm{NMR}\left(100 \mathrm{MHz}, \mathrm{CDCl}_{3}\right): \delta=162.1,141.2,138.7,132.3,128.6,127.2$, 126.6, 125.3, 124.1, 121.6, 110.7, 76.2, 68.1, 34.3, 26.0; IR (KBr): 3348, 2956, 1652, 1604, 1480, 1286, 1218, 1152, 882, 764, $698 \mathrm{~cm}^{-1}$; HRMS (FAB): $\mathrm{m} / z$ calcd for $\mathrm{C}_{38} \mathrm{H}_{40} \mathrm{~N}_{3} \mathrm{O}_{2} 570.3121$, found 570.3137.

\section{1,8-bis((S)-4-methyl-4,5-dihydrooxazol-2-yl)-3,6-diphenyl-9H-carbazole (1a)}

Crude 1,8-dicyano-3,6-diphenyl carbazole prepared from 1,8-diiodo-3,6-diphenyl carbazole (2) (1.53 g, $2.68 \mathrm{mmol})$ and $\mathrm{CuCN}(0.682 \mathrm{~g}, 7.62 \mathrm{mmol})$ as described above was suspended in $\mathrm{PhCl}(20 \mathrm{ml})$, and to this suspension were added L-alaninol $(0.63 \mathrm{ml}, 8.09 \mathrm{mmol})$ and $\mathrm{ZnCl}_{2}(1.08 \mathrm{~g}, 7.92 \mathrm{mmol})$ under Ar. The reaction mixture was refluxed for 3 days, and then was cooled to ambient temperature. After the addition of a solution of ethylenediamine $(5 \mathrm{ml})$ in $\mathrm{H}_{2} \mathrm{O}(50 \mathrm{ml})$, the mixture was stirred for $1 \mathrm{~h}$. The resulting mixture was extracted with $\mathrm{CH}_{2} \mathrm{Cl}_{2}(50 \mathrm{ml}$ x 4$)$, and the combined organic layer was washed with brine ( $50 \mathrm{ml}$ ), dried over $\mathrm{Na}_{2} \mathrm{SO}_{4}$, evaporated. The residue was dissolved in THF (30 ml), and to this solution was added a mixture of $\mathrm{AcOH}(10 \mathrm{ml}), \mathrm{H}_{2} \mathrm{O}(2 \mathrm{ml})$ and $\mathrm{H}_{2} \mathrm{SO}_{4}(0.3 \mathrm{ml})$. The solution immediately was stirred for $1 \mathrm{~h}$ at room temperature. The resulting mixture was extracted with ethyl 
acetate $\left(50 \mathrm{ml} \mathrm{x} \mathrm{4)}\right.$, and organic layer was wash with brine (50 ml), dried over $\mathrm{Na}_{2} \mathrm{SO}_{4}$, evaporated. The residue was purified by flash chromatography (hexane/ethyl acetate=6/1-4/1). Recrystallization from hexane/ethyl acetate afforded white crystalline ligand $\mathbf{1 a}(0.595 \mathrm{~g}, 46 \%, 3$ steps from 2$)$ : $[\alpha]_{\mathrm{D}}^{23}-8.31$ (c 1.19, $\mathrm{CHCl}_{3}$ ); mp 182-183 ${ }^{\circ} \mathrm{C} ;{ }^{1} \mathrm{H}$ NMR (400 MHz, $\left.\mathrm{CDCl}_{3}\right): \delta=11.9$ (s, $\left.1 \mathrm{H}\right), 8.44(\mathrm{~d}, J=1.5 \mathrm{~Hz}, 2 \mathrm{H})$, 8.19 (d, $J=1.5 \mathrm{~Hz}, 2 \mathrm{H}$ ), 7.75 (dddd, $J=7.3,1.7,1.7,1.2 \mathrm{~Hz}, 4 \mathrm{H}$ ), 7.47 (dddd, $J=7.3,7.3,1.7,1.2 \mathrm{~Hz}$, $4 \mathrm{H}), 7.34(\mathrm{tt}, J=7.3,1.7 \mathrm{~Hz}, 2 \mathrm{H}), 4.63(\mathrm{dd}, J=9.3,7.1 \mathrm{~Hz}, 2 \mathrm{H}), 4.57$ (ddq, $J=9.3,6.8,6.3 \mathrm{~Hz}, 2 \mathrm{H}), 4.03$ (dd, $J=7.1,6.8 \mathrm{~Hz}, 2 \mathrm{H}), 1.51(\mathrm{~d}, J=6.3 \mathrm{~Hz}, 6 \mathrm{H}) ;{ }^{13} \mathrm{C} \mathrm{NMR}\left(100 \mathrm{MHz}, \mathrm{CDCl}_{3}\right): \delta=162.5,141.3,138.7$, 132.4, 128.7, 127.2, 126.6, 125.1, 124.1, 121.8, 110.7, 73.6, 62.3, 21.7; IR (KBr): 3348, 2972, 1644, $1602,1480,1290,1218,1160,1054,994,880,762,698 \mathrm{~cm}^{-1}$; HRMS (FAB): $m / z$ calcd for $\mathrm{C}_{32} \mathrm{H}_{28} \mathrm{~N}_{3} \mathrm{O}_{2}$ 486.2182 , found 486.2180 .

\section{General procedure for the enantioselective propargylation with 1c-Cr complex.}

\section{(R)-1-phenylbut-3-yn-1-ol (2)}

A mixture of $1 \mathbf{c}(34.0 \mathrm{mg}, 0.0597 \mathrm{mmol})$ and $\mathrm{CrCl}_{2}(6.2 \mathrm{mg}, 0.050 \mathrm{mmol})$ was azeotroped with toluene and dried under high vacuum, and was dissolved in THF ( $2 \mathrm{ml})$ under Ar atmosphere. The color turned to deep brown gradually. After stirring at $60^{\circ} \mathrm{C}$ for $12 \mathrm{~h}$, to the mixture was added DIPEA $(0.026 \mathrm{ml}$, $0.15 \mathrm{mmol})$. After stirring at $60^{\circ} \mathrm{C}$ for additional $1 \mathrm{~h}, \mathrm{H}_{2} \mathrm{O}(0.1 \mathrm{ml})$ was added in open air. The mixture was filtered through Celite, and evaporated in vacuo. To the residue was added $\mathrm{H}_{2} \mathrm{O}(15 \mathrm{ml})$ and $\mathrm{CH}_{2} \mathrm{Cl}_{2}(15 \mathrm{ml})$, and the aqueous layer was extracted with $\mathrm{CH}_{2} \mathrm{Cl}_{2}(15 \mathrm{ml} \times 3)$. The combined organic layer was washed with brine $(20 \mathrm{ml})$, dried over $\mathrm{MgSO}_{4}$, evaporated. A mixture of the crude complex and $\mathrm{Mn}$ (56.7 mg, $1.03 \mathrm{mmol}$ ) was azeotroped with toluene, and was suspended in DME (2 ml). After stirring $15 \mathrm{~min}$, to the suspension were added DIPEA $(0.026 \mathrm{ml}, 0.15 \mathrm{mmol})$ and propargylbromide $(0.075 \mathrm{ml}, 1.0 \mathrm{mmol})$. After $5 \mathrm{~min}$, to the mixture was added benzaldehyde $(0.050 \mathrm{ml}, 0.49 \mathrm{mmol})$, TMSCl $(0.125 \mathrm{ml}, 0.98 \mathrm{mmol})$ successively, and the resulting mixture was stirred for $12 \mathrm{~h}$ at room temperature. The reaction was quenched with saturated aqueous $\mathrm{NaHCO}_{3}(0.5 \mathrm{ml})$, filtered through Celite, and evaporated. The crude product was dissolved in THF ( $2 \mathrm{ml})$, and the stirred mixture was treated with TBAF (1.0 ml, $1 \mathrm{M}$ solution). The reaction was quenched with saturated aqueous $\mathrm{NH}_{4} \mathrm{Cl}$ (2 $\mathrm{ml})$, extracted with $\mathrm{Et}_{2} \mathrm{O}\left(10 \mathrm{ml}\right.$ x 4), dried over $\mathrm{Na}_{2} \mathrm{SO}_{4}$, and evaporated. The residue was purified by flash chromatography (hexane/ethyl acetate=8/1) to afford the known compound, (R)-1-phenylbut-3-yn-1-ol (66.4 mg, 93\%, 78\% ee): $[\alpha]_{\mathrm{D}}{ }^{28}+11.1(\mathrm{c} 0.71, \mathrm{MeOH}),\left(\right.$ lit. $^{15 \mathrm{~h}}[\alpha]_{\mathrm{D}}+12.9(\mathrm{c}$ 1.55, MeOH)); ee was determined by HPLC; Daicel Chiral Pack OD-H $0.46 \mathrm{~cm} \phi \times 25 \mathrm{~cm}$; hexane/isopropanol=19/1; flow rate=0.5 $\mathrm{ml} / \mathrm{min}$ ); retention time: $20.4 \mathrm{~min}$ for 
(R)-1-phenylbut-3-yn-1-ol, 24.1 min for (S)-1-phenylbut-3-yn-1-ol.

\section{(R)-1-(naphthalen-2-yl)but-3-yn-1-ol (3)}

$[\alpha]_{\mathrm{D}}^{19}-1.4(\mathrm{c} 0.57, \mathrm{MeOH}),\left(\right.$ lit. $^{15 \mathrm{~h}}[\alpha]_{\mathrm{D}}{ }^{24}-2.67$ (c 1.16, EtOH)); ee was determined by HPLC; Daicel Chiral Pack AS-H $0.46 \mathrm{~cm} \phi \times 25 \mathrm{~cm}$; hexane/isopropanol=3/1; flow rate $=0.2 \mathrm{ml} / \mathrm{min}$ ); retention time: $40.2 \mathrm{~min}$ for (R)-1-(naphthalen-2-yl)but-3-yn-1-ol, $44.4 \mathrm{~min}$ for (S)-1-(naphthalen-2-yl)but-3-yn-1-ol.

\section{(R)-(E)-1-phenylhex-1-en-5-yn-3-ol (4)}

$[\alpha]_{D}{ }^{24}-11.1$ (c 1.50, benzene), (lit. ${ }^{15 \mathrm{~h}}[\alpha]_{\mathrm{D}}{ }^{24}-59.27$ (c 1.35, benzene)); ee was determined by HPLC; Daicel Chiral Pack OD-H $0.46 \mathrm{~cm} \phi \times 25 \mathrm{~cm}$; hexane/isopropanol=19/1; flow rate=0.5 ml/min); retention time: $33.7 \mathrm{~min}$ for $(R, E)$-1-phenylhex-1-en-5-yn-3-ol, $48.6 \mathrm{~min}$ for (S)-(E)-1-phenylhex-1-en-5-yn-3-ol.

\section{(S)-1-phenylhex-5-yn-3-ol (5)}

$[\alpha]_{\mathrm{D}}{ }^{20}-11.6\left(\mathrm{c} 0.89, \mathrm{CHCl}_{3}\right),\left((R)\right.$-form; lit. ${ }^{12}[\alpha]_{\mathrm{D}}{ }^{20}+3.5\left(\mathrm{c} 0.56, \mathrm{CHCl}_{3}\right)$ ); ee was determined by HPLC; Daicel Chiral Pack OD-H $0.46 \mathrm{~cm} \phi \times 25 \mathrm{~cm}$; hexane/isopropanol=19/1; flow rate=0.5 $\mathrm{ml} / \mathrm{min}$ ); retention time: $25.5 \mathrm{~min}$ for $(R)$-1-phenylhex-5-yn-3-ol, $39.4 \mathrm{~min}$ for $(S)$ -1-phenylhex-5-yn-3-ol.

\section{(S)-non-1-yn-4-ol (6)}

$[\alpha]_{\mathrm{D}}{ }^{24}+2.2(\mathrm{c} 0.4, \mathrm{MeOH}),\left(\right.$ lit. $\left.^{15 \mathrm{j}}[\alpha]_{\mathrm{D}}{ }^{28}+22.2(\mathrm{c} 1.0, \mathrm{MeOH})\right)$

\section{(S)-non-1-yn-4-yl 3,5-dinitrobenzoate}

${ }^{1} \mathrm{H} \mathrm{NMR}\left(400 \mathrm{MHz}, \mathrm{CDCl}_{3}\right): \delta=9.24(\mathrm{t}, J=2.2 \mathrm{~Hz}, 1 \mathrm{H}), 9.18(\mathrm{~d}, J=2.2 \mathrm{~Hz}, 2 \mathrm{H}), 5.31$ (dddd, $J=7.6,5.9$, 5.6, 5.4 Hz, 1H), 2.69 (ddd, J=17.1, 5.6, $2.7 \mathrm{~Hz}, 1 \mathrm{H}$ ), 2.65 (ddd, J=17.1, 5.9, 2.7 Hz, 1H), 2.04 (dd, $J=2.7,2.7 \mathrm{~Hz}, 1 \mathrm{H}), 1.95-1.81(\mathrm{~m}, 2 \mathrm{H}), 1.45-1.24(\mathrm{~m}, 6 \mathrm{H}), 0.90(\mathrm{t}, J=6.8 \mathrm{~Hz}, 3 \mathrm{H})$; ee was determined by HPLC; Daicel Chiral Pack OD-H $0.46 \mathrm{~cm} \phi \times 25 \mathrm{~cm}$; hexane/isopropanol=19/1; flow rate=0.5 $\mathrm{ml} / \mathrm{min}$ ); retention time: $33.6 \mathrm{~min}$ for $(R)$-non-1-yn-4-yl 3,5-dinitrobenzoate, $40.6 \mathrm{~min}$ for (S)-non-1-yn-4-yl 3,5-dinitrobenzoate.

\section{(R)-1-cyclohexylbut-3-yn-1-ol (7)}

$[\alpha]_{\mathrm{D}}{ }^{25}+9.50\left(\mathrm{c} 0.56, \mathrm{CHCl}_{3}\right),\left(\mathrm{lit}^{15 \mathrm{i}}[\alpha]_{\mathrm{D}}+9.7\left(\mathrm{c} 1.0, \mathrm{CHCl}_{3}\right)\right)$.

\section{(R)-1-cyclohexylbut-3-ynyl 3,5-dinitrobenzoate}


${ }^{1} \mathrm{H} \mathrm{NMR}\left(400 \mathrm{MHz}, \mathrm{CDCl}_{3}\right.$ ): $\delta=9.24(\mathrm{t}, J=2.2 \mathrm{~Hz}, 1 \mathrm{H}), 9.17$ (d, $\left.J=2.2 \mathrm{~Hz}, 2 \mathrm{H}\right), 5.15$ (ddd, $J=6.6,6.3$, $5.1 \mathrm{~Hz}, 1 \mathrm{H}), 2.69$ (ddd, J=17.3, 5.1, $2.7 \mathrm{~Hz}, 1 \mathrm{H}), 2.66$ (ddd, J=17.3, 6.3, $2.7 \mathrm{~Hz}, 1 \mathrm{H}$ ), 1.99 (dd, $J=2.7,2.7 \mathrm{~Hz}, 1 \mathrm{H}), 1.95-1.69(\mathrm{~m}, 6 \mathrm{H}), 1.38-1.01$ (m, 5H); ee was determined by HPLC; Daicel Chiral Pack AS-H $0.46 \mathrm{~cm} \quad \phi \times 25 \mathrm{~cm}$; hexane/isopropanol=6/1; flow rate=0.3 ml/min); retention time: $30.2 \mathrm{~min}$ for $(R)$-1-cyclohexylbut-3-ynyl 3,5-dinitrobenzoate, $43.8 \mathrm{~min}$ for (S)-1-cyclohexylbut-3-ynyl 3,5-dinitrobenzoate.

\section{(R)-2,2-dimethylhex-5-yn-3-ol (8)}

$[\alpha]_{\mathrm{D}}{ }^{24}+19.8(\mathrm{c} 0.60, \mathrm{MeOH}),\left((S)\right.$-form; lit. $\left.^{15 \mathrm{k}}[\alpha]_{\mathrm{D}}{ }^{25}-38.28(\mathrm{c} 3.6, \mathrm{MeOH})\right)$.

\section{(R)-MTPA ester of (R)-2,2-dimethylhex-5-yn-3-ol ${ }^{151}$}

${ }^{1} \mathrm{H}$ NMR (400 MHz, $\mathrm{CDCl}_{3}$ ): $\delta=7.69-7.66(\mathrm{~m}, S-2 \mathrm{H}), 7.64-7.61(\mathrm{~m}, R-2 \mathrm{H}), 7.42-7.37(\mathrm{~m}, 6 \mathrm{H}), 5.14$ (dd, $J=9.0,3.4 \mathrm{~Hz}, S-1 \mathrm{H}), 5.12$ (dd, $J=9.0,3.7 \mathrm{~Hz}, R-1 \mathrm{H}$ ), 3.63 (dd, $J=2.4,1.0 \mathrm{~Hz}, S-3 \mathrm{H}$ ), 3.53 (dd, $J=1.7,1.0 \mathrm{~Hz}, R-3 \mathrm{H}$ ), 2.62 (ddd, $J=15.9,3.4,2.7 \mathrm{~Hz}, S-1 \mathrm{H}$ ), 2.58 (ddd, $J=17.1,3.7,2.7 \mathrm{~Hz}, R-1 \mathrm{H}$ ), 2.47 (ddd, $J=15.9,9.0,2.7 \mathrm{~Hz}, S-1 \mathrm{H}$ ), 2.44 (ddd, $J=17.1,9.0,2.7 \mathrm{~Hz}, R-1 \mathrm{H}$ ), 1.97 (dd, $J=2.7,2.7 \mathrm{~Hz}$, $S-1 \mathrm{H}), 1.86$ (dd, J=2.7, $2.7 \mathrm{~Hz}, R-1 \mathrm{H}), 0.95(\mathrm{~s}, R-9 \mathrm{H}), 0.88$ (s, $S-9 \mathrm{H})$.

15) (h) Denmark, S. E.; Wynn, T. J. Am. Chem. Soc. 2001, 123, 6199-6200.

(i) Ikeda, N.; Omori, K.; Yamamoto, H. Tetrahedron Lett. 1986, 27, 1175-1178.

(j) Takano, S.; Setoh, M.; Ogasawara, K. Tetrahedron: Asymmetry 1992, 3, 533-534.

(k) Haruta, R.; Ishiguro, M.; Ikeda, N., Yamamoto, H. J. Am. Chem. Soc. 1982, 104, 7667-7669.

(1) Corey, E. J.; Yu, C.; Lee, D. J. Am. Chem. Soc. 1990, 112, 878-879. 